\title{
MINIMAL COEFFICIENTS IN HÖLDER CONDITIONS IN THE WIENER SPACE ${ }^{1}$
}

\author{
J. YEH
}

Abstract. For almost every $x$ in the Wiener space $C_{w}$, the Hölder condition $\left|x\left(t^{\prime}\right)-x\left(t^{\prime \prime}\right)\right| \leqq h\left|t^{\prime}-t^{\prime \prime}\right| \alpha$ holds for some $h>0$ when $\alpha \in\left(0, \frac{1}{2}\right)$. Let $\phi_{\alpha}[x]$ be the infimum of all $h>0$ for fixed $x$ and $\alpha$. In the present paper we prove that every positive power of $\phi_{\alpha}[x]$ is Wiener integrable over $C_{w}$ and give an estimate for the Wiener integral.

1. Introduction. Let $C_{w}$ be the Wiener space consisting of all real valued continuous functions $x(t), t \in T=[0,1]$, with $x(0)=0 . \mathrm{N}$. Wiener [2] showed that for every $\alpha \in\left(0, \frac{1}{2}\right)$ almost every $x \in C_{w}$ satisfies the Hölder condition $\left|x\left(t^{\prime}\right)-x\left(t^{\prime \prime}\right)\right| \leqq h\left|t^{\prime}-t^{\prime \prime}\right| \alpha$ for some $h>0$ which depends on $x$ and $\alpha$. Let $\phi_{\alpha}[x]$ be the infimum of all such $h>0$ for fixed $x$ and $\alpha$. Our result is the following

Theorem. For every $\alpha \in\left(0, \frac{1}{2}\right)$, the functional $\left\{\phi_{\alpha}[x]\right\}^{p}$ is Wiener integrable for all $p>0$, i.e. $\int_{C_{w}}\left\{\phi_{\alpha}[x]\right\}^{p} d_{w} x<\infty$. In fact

$$
\begin{aligned}
& \int_{C_{w}}\left\{\phi_{\alpha}[x]\right\}^{p} d_{w} x \leqq\left(\frac{2}{1-2^{-\alpha}}\right)^{p} \\
& \quad\left[1+\left(\frac{2}{\pi}\right)^{1 / 2}(2 N)^{N} e^{-N}\left\{1-2^{1 / 2+\alpha-N(1-2 \alpha)}\right\}^{-1} \sum_{m=1}^{\infty} \frac{(m+1)^{p}}{m^{2 N+1}}\right] \\
& \quad<\infty
\end{aligned}
$$

for every positive number $N$ such that

$$
N>\frac{1}{2} \max \left\{\frac{1+2 \alpha}{1-2 \alpha}, p\right\} .
$$

We think that the above result might be applied in establishing the convergence of Wiener integrals or in estimating errors in approximating Wiener integrals by ordinary Lebesgue integrals on finite dimensional Euclidean spaces. The theorem will be proved in $\$ 3$.

Received by the editors September 19, 1969.

AMS Subject Classifications. Primary 2846; Secondary 2825, 6062.

Key Words and Phrases. Wiener measure, Brownian motion, continuity of sample paths, Hölder condition, essential boundedness.

1 This research was supported in part by National Science Foundation Grant GP-5436. 
2. Estimates of Wiener measures of various subsets of $C_{w}$. Let $D$ be the collection of binary rationals in $T$. According to $N$. Wiener, for every fixed $\alpha \in\left(0, \frac{1}{2}\right)$ almost every $x \in C_{w}$ satisfies the Hölder condition $\left|x\left(t^{\prime}\right)-x\left(t^{\prime \prime}\right)\right| \leqq h\left|t^{\prime}-t^{\prime \prime}\right| \alpha, t^{\prime}, t^{\prime \prime} \in D$, where $h>0$ depends on $x$.

Definition 1 . For every $\alpha>0$ and $h>0$ let $H_{\alpha}[h]$ consist of those functions $x \in C_{w}$ for which there exist $t^{\prime}, t^{\prime \prime} \in D$, depending on $x$, satisfying the inequality $\left|x\left(t^{\prime}\right)-x\left(t^{\prime \prime}\right)\right|>h\left|t^{\prime}-t^{\prime \prime}\right| \alpha$.

$H_{\alpha}[h]$, as the complement of a Wiener measurable set, is itself Wiener measurable.

Lemma 1. Let $\alpha>0$ and $h>0$. If $x \in C_{w}$ satisfies

$$
\left|x\left(n 2^{-k}\right)-x\left((n-1) 2^{-k}\right)\right| \leqq h 2^{-k \alpha}
$$

for every nonnegative integer $k$ and positive integer $n, 1 \leqq n \leqq 2^{k}$, then $x \notin H_{\alpha}\left[2 h\left(1-2^{-\alpha}\right)^{-1}\right]$.

Proof. Each $r \in D$, being a binary rational in $T$, can be expressed as $q 2^{-p}$ where $p$ and $q$ are nonnegative integers and $p$ is chosen to be the smallest possible for each $r$. Thus for $r=0$ we have $p=0, q=0$ and for $r=1$ we have $p=0, q=1$. These are the only two for which $p=0$. Also, when $r \neq 0, q$ is odd.

Let $r_{1}, r_{2} \in D, r_{1}<r_{2}$. Consider the case $\left[r_{1}, r_{2}\right] \neq[0,1]$. Let $r_{3} \in\left[r_{1}, r_{2}\right]$ be the binary rational with the smallest $p$ among all the binary rationals in $\left[r_{1}, r_{2}\right]$. There is exactly one such $r_{3}$. For if one of $r_{1}$ and $r_{2}$ is 0 or 1 then $r_{3}$ is the one with $p=0$; if none of $r_{1}$ and $r_{2}$ is 0 or 1 and there were two distinct $r_{3}$, say $q_{1} 2^{-p}$ and $q_{2} 2^{-p}$, then the choice of $r_{3}$ would be contradicted since there would be an even integer between $q_{1}$ and $q_{2}$. When $\left[r_{1}, r_{2}\right]=[0,1]$, we let $r_{3}=r_{1}$.

Now if $r_{3} \neq r_{1}$, then $r_{3}-r_{1}=\sum_{i=1}^{l} 2^{-k_{i}}$ and if $r_{3} \neq r_{2}$ we have $r_{2}-r_{3}$ $=\sum_{j=1}^{m} 2^{-l_{j}}$ where $\left\{k_{i}\right\}$ and $\left\{l_{j}\right\}$ are strictly increasing finite sequences of nonnegative integers. Consider the two sequences of intervals:

$$
\begin{array}{r}
{\left[r_{3}-2^{-k_{1}}, r_{3}\right],\left[r_{3}-2^{-k_{1}}-2^{-k_{2}}, r_{3}-2^{-k_{1}}\right], \cdots,} \\
{\left[r_{1}, r_{3}-2^{-k_{1}}-\cdots-2^{-k_{l-1}}\right]}
\end{array}
$$

and

$$
\begin{aligned}
{\left[r_{3}, r_{3}+2^{-j_{1}}\right],\left[r_{3}+2^{-l_{1}}, r_{3}+2^{-l_{1}}+2^{-l_{2}}\right], \cdots, } \\
\\
{\left[r_{3}+2^{-l_{1}}+\cdots+2^{-l_{m-1}}, r_{2}\right] . }
\end{aligned}
$$

Since $r_{1}, r_{2}$ and $r_{3}$ cannot be all equal, at least one of the two sequences 
of subintervals of $\left[r_{1}, r_{2}\right]$ exists and $\left[r_{1}, r_{2}\right]$ is decomposed into those subintervals that exist.

Let $p=\min \left\{k_{1}, l_{1}\right\}, q=\max \left\{k_{l}, l_{m}\right\}$. By $(2.1)$

$$
\begin{aligned}
\left|x\left(r_{1}\right)-x\left(r_{2}\right)\right| & \leqq 2 h \sum_{k=p}^{q} 2^{-k \alpha} \leqq 2 h 2^{-p \alpha}\left(1-2^{-\alpha}\right)^{-1} \\
& \leqq 2 h\left(1-2^{-\alpha}\right)^{-1}\left|r_{1}-r_{2}\right|^{\alpha} .
\end{aligned}
$$

Since this is true of every pair $r_{1}, r_{2} \in D, x \notin H_{\alpha}\left[2 h\left(1-2^{-\alpha}\right)^{-1}\right]$.

Lemma 2. Let $\alpha>0$ and $h>0$. Then (1) for the Wiener measure $m_{w}$ we have

$$
m_{w}\left(H_{\alpha}\left[2 h\left(1-2^{-\alpha}\right)^{-1}\right]\right)
$$

$$
\leqq\left(\frac{2}{\pi}\right)^{1 / 2} \frac{1}{h} \sum_{k=0}^{\infty} 2^{k(1 / 2+\alpha)} \exp \left\{-\frac{h^{2}}{2} 2^{k(1-2 \alpha)}\right\} .
$$

(2) The series in the right-hand side of (2.2) (not including the factor $\left.(2 / \pi)^{1 / 2}(1 / h)\right)$ diverges for $\alpha \geqq \frac{1}{2}$ and converges for $0<\alpha<\frac{1}{2}$ to a sum which decreases monotonically with increasing $h$.

Proof. (1). Let $\alpha>0, h>0$ and $x \in H_{\alpha}\left[2 h\left(1-2^{-\alpha}\right)^{-1}\right]$. According to Lemma 1

$$
x \in I_{\alpha, h, k, n}=\left\{x \in C_{w} ;\left|x\left(n 2^{-k}\right)-x\left((n-1) 2^{-k}\right)\right|>h 2^{-k \alpha}\right\}
$$

for some nonnegative integer $k$ and positive integer $n, 1 \leqq n \leqq 2^{k}$, and consequently

$$
H_{\alpha}\left[2 h\left(1-2^{-\alpha}\right)^{-1}\right] \subset \bigcup_{k=0}^{\infty} \bigcup_{n=1}^{2^{k}} I_{\alpha, h, k, n}
$$

Now by the definition of the Wiener measure

$$
\begin{aligned}
m_{w}\left(I_{\alpha, h, k, n}\right)= & \left\{(2 \pi)^{2}(n-1) 2^{-k} 2^{-k}\right\}^{-1 / 2} \\
& \cdot \int_{\left|\xi_{1}-\xi_{2}\right|>h 2^{-k d}} \exp \left\{-\frac{2^{k} \xi_{1}^{2}}{2(n-1)}-\frac{2^{k}\left(\xi_{2}-\xi_{1}\right)^{2}}{2}\right\} d \xi_{1} d \xi_{2} .
\end{aligned}
$$

Letting $\xi=\left\{2(n-1) 2^{-k}\right\}^{-1 / 2} \xi_{1}, \eta=\left\{2 \cdot 2^{-k}\right\}^{-1 / 2}\left(\xi_{2}-\xi_{1}\right)$ and applying the inequality

$$
\int_{\lambda}^{\infty} \exp \left[-\xi^{2}\right] d \xi \leqq \frac{1}{2 \lambda} \exp \left[-\lambda^{2}\right] \quad \text { for } \lambda>0
$$


we obtain

$$
\begin{aligned}
m_{w}\left(I_{\alpha, h, k, n}\right) & =\frac{1}{\pi}\left\{\int_{-\infty}^{\infty} \exp \left[-\xi^{2}\right] d \xi\right\}\left\{2 \int_{h 2^{-1 / 2} 2^{k(1 / 2-\alpha)}}^{\infty} \exp \left[-\eta^{2}\right] d \eta\right\} \\
& \leqq\left(\frac{2}{\pi}\right)^{1 / 2} \frac{1}{h} 2^{k(\alpha-1 / 2)} \exp \left\{-\frac{h^{2}}{2} 2^{k(1-2 \alpha)}\right\} .
\end{aligned}
$$

Thus

$$
\begin{aligned}
m_{w}\left(H_{\alpha}\left[2 h\left(1-2^{-\alpha}\right)^{-1}\right]\right) & \leqq \sum_{k=0}^{\infty} \sum_{n=1}^{2^{k}} m_{w}\left(I_{\alpha, h, k, n}\right) \\
& =\left(\frac{2}{\pi}\right)^{1 / 2} \frac{1}{h} \sum_{k=0}^{\infty} 2^{k(1 / 2+\alpha)} \exp \left\{-\frac{h^{2}}{2} 2^{k(1-2 \alpha)}\right\} .
\end{aligned}
$$

(2) follows from (2.2).

\section{3 . Proof of the theorem.}

Definition 2. Let $\alpha>0$ be fixed. For each $x \in C_{w}$ for which there exists some $h>0$ satisfying $\left|x\left(t^{\prime}\right)-x\left(t^{\prime \prime}\right)\right| \leqq h\left|t^{\prime}-t^{\prime \prime}\right| \alpha$ for all $t^{\prime}, t^{\prime \prime} \in D$ we define $\phi_{\alpha}[x]=\inf h$ where the infimum is taken over the set of all such positive numbers $h$. If no such $h$ exists for $x$ we define $\phi_{\alpha}[x]=\infty$.

REMARK 1. For every $x \in C_{w},\left|x\left(t^{\prime}\right)-x\left(t^{\prime \prime}\right)\right| \leqq \phi_{\alpha}[x]\left|t^{\prime}-t^{\prime \prime}\right|^{\alpha}$ for all $t^{\prime}, t^{\prime \prime} \in D$.

REMARK 2. $0 \leqq \phi_{\alpha}[x] \leqq \infty$ for $x \in C_{w}$. In fact $x(t) \equiv 0$ is the only function in $C_{w}$ for which $\phi_{\alpha}[x]=0$. When $0<\alpha<\frac{1}{2}, \phi_{\alpha}[x]<\infty$ for almost every $x \in C_{w}$.

REMARK 3. For every $h>0$,

$$
\begin{aligned}
\left\{x \in C_{w} ; \phi_{\alpha}[x]\right. & \leqq h\} \\
& =\left\{x \in C_{w} ;\left|x\left(t^{\prime}\right)-x\left(t^{\prime \prime}\right)\right| \leqq h\left|t^{\prime}-t^{\prime \prime}\right|^{\alpha}, t^{\prime}, t^{\prime \prime} \in D\right\} .
\end{aligned}
$$

Since the right-hand side of this equality is a Wiener measurable set, $\phi_{\alpha}[x]$ is a Wiener measurable function.

REMARK 4. $x \in H_{\alpha}[h]$ if and only if $\phi_{\alpha}[x]>h$.

Proof of THE Theorem. Decompose $C_{w}$ into a sequence of disjoint Wiener measurable sets given by

$$
\begin{aligned}
\Gamma_{0} & =\left\{x \in C_{w} ; \phi_{\alpha}[x] \leqq 2\left(1-2^{-\alpha}\right)^{-1}\right\}, \\
\Gamma_{m}=\left\{x \in C_{w} ; 2 m\left(1-2^{-\alpha}\right)^{-1}<\phi_{\alpha}[x] \leqq 2(m+1)\left(1-2^{-\alpha}\right)^{-1}\right\}, & m=1,2, \cdots .
\end{aligned}
$$

By Remark 4 and (2.2) 


$$
\begin{aligned}
\left.\int_{C_{w}}\left\{\phi_{\alpha}[x]\right\}\right\}^{p} d_{w} x= & \sum_{m=0}^{\infty} \int_{\Gamma_{m}}\left\{\phi_{\alpha}[x]\right\}^{p} d_{w} x \\
\leqq & 2^{p}\left(1-2^{-\alpha}\right)^{-p}+\sum_{m=1}^{\infty} 2^{p}(m+1)^{p}\left(1-2^{-\alpha}\right)^{-p} m_{w}\left(I_{m}\right) \\
\leqq & 2^{p}\left(1-2^{-\alpha}\right)^{-p}+2^{p}\left(1-2^{-\alpha}\right)^{-p}\left(\frac{2}{\pi}\right)^{1 / 2} \\
& \cdot \sum_{m=1}^{\infty}(m+1)^{p} \frac{1}{m} \sum_{k=0}^{\infty} 2^{k(1 / 2+\alpha)} \exp \left\{-\frac{m^{2}}{2} 2^{k(1-2 \alpha)}\right\} .
\end{aligned}
$$

It remains to show that the double sum in the last member of the above inequalities is finite whenever $\alpha \in\left(0, \frac{1}{2}\right)$ and $p>0$. Now for every positive number $N$ the function $x^{N} \exp \left[-\left(m^{2} / 2\right) x\right], x>0$, assumes its maximum value when $x=2 N / m^{2}$. Thus $x^{N} \exp \left[-\left(m^{2} / 2\right) x\right]$ $\leqq\left(2 N / e m^{2}\right)^{N}$ for $x>0$ and therefore

$$
\exp \left\{-\left(m^{2} / 2\right) \cdot 2^{k(1-2 \alpha)}\right\} \leqq 2^{-k N(1-2 \alpha)}\left(2 N / e m^{2}\right)^{N}
$$

for each $m>0$ and each $k \geqq 0$. Using this we obtain

$$
\begin{aligned}
\sum_{m=1}^{\infty}(m+1)^{p} \frac{1}{m} \sum_{k=0}^{\infty} 2^{k(1 / 2+\alpha)} & \exp \left\{-\frac{m^{2}}{2} 2^{k(1-2 \alpha)}\right\} \\
& \leqq \sum_{m=1}^{\infty}(m+1)^{p} \frac{1}{m}\left(\frac{2 N}{e m^{2}}\right)^{N} \sum_{k=0}^{\infty} 2^{k\{1 / 2+\alpha-N(1-2 \alpha)\}}
\end{aligned}
$$

Now choose $N$ so that (1.2) is satisfied. Then $\frac{1}{2}+\alpha-N(1-2 \alpha)<0$ and therefore

$$
\sum_{k=0}^{\infty} 2^{k\{1 / 2+\alpha-N(1-2 \alpha)\}}=\left\{1-2^{1 / 2+\alpha-N(1-2 \alpha)}\right\}^{-1}<\infty .
$$

Thus we get

$$
\begin{aligned}
\sum_{m=1}^{\infty}(m+1)^{p} \frac{1}{m}\left(\frac{2 N}{e m^{2}}\right)^{N} & \sum_{k=0}^{\infty} 2^{k\{1 / 2+\alpha-N(1-2 \alpha)\}} \\
& \leqq(2 N)^{N} e^{-N}\left\{1-2^{1 / 2+\alpha-N(1-2 \alpha)}\right\}-1 \sum_{m=1}^{\infty} \frac{(m+1)^{p}}{m^{2 N+1}} \\
& <\infty
\end{aligned}
$$

since $2 N>p$ by (1.2). This completes the proof. 


\section{BIBLIOGRAPHY}

1. I. M. Gel'fand and A. M. Jaglom, Integration in function spaces and its application to quantum physics, Uspehi Mat. Nauk 11 (1956), no. 1 (67), 77-114. (Russian) MR 17, 1261.

2. N. Wiener, Generalized harmonic analysis, Acta Math 55 (1940), 117-258.

3. J. Yeh, Wiener measure in a space of functions of two variables, Trans. Amer. Math. Soc. 95 (1960), 433-450. MR 23 \#A2735.

University of California, Irvine, California 92664 